\title{
Osteotomien bei knöcherner Infektion
}

\author{
G. O. Hofmann
}

\section{Zusammenfassung}

Osteotomien bei knöchernen Infektionen müssen vor allem dann durchgeführt werden, wenn es um die Resektion von Knochensegmenten geht, die von chronischer Osteitis erfasst sind. Dabei ergibt sich die Indikation zur Osteotomie in den allermeisten Fällen nicht nur aus der Notwendigkeit einer Stellungskorrektur, sondern auch aus der Beseitigung eines zwar knöchern stabilen, jedoch chronisch infizierten Knochenareals oder einer Infekt-Pseudarthrose. Da es sich hierbei um operative Eingriffe aus dem Bereich der septischen Knochenchirurgie handelt, müssen andere Behandlungsgrundsätze gelten als bei aseptischen Korrekturosteotomien und Umstellungen. Im folgenden Beitrag soll das von uns gewählte Vorgehen, aufgegliedert in einzelne Behandlungsetappen, vorgestellt werden.

\section{Einleitung}

Eine chronische Osteitis steht bedauerlicherweise oft als Endresultat eines komplizierten Behandlungsverlaufes nach Frakturen an langen Röhrenknochen. Dieser Endzustand kann in verschiedenen Formen vorliegen:

- chronischer Infekt bei stellungskorrekter knöcherner Ausheilung

- chronischer Infekt bei knöcherner Konsolidierung in Fehlstellung

- infizierte Pseudarthrose

Die Frage, ob eine permanente oder temporäre Fistel besteht, ob noch Osteosyn-

OP-JOURNAL 2000; 16: 79-82

(c) Georg Thieme Verlag Stuttgart · New York theseimplantate als Fremdkörper einliegen, ist dabei völlig sekundär. Sieht man von den wenigen Fällen $a b$, bei denen in Ausnahmesituationen (hohes Alter, moribunder Patient, rezidivierende Therapieresistenz) eine chronische Fistel als natürliche Sekretdrainage toleriert wird, erfolgt die Behandlung nach einem standardisierten Vorgehen. Wir gliedern dabei den therapeutischen Ablauf in 3 Phasen:

- Phase 1: Infektsanierung

- Phase 2: Rekonstruktion des

Haut-/Weichteilmantels

- Phase 3: Überbrückung der knöchernen Defektstrecke

Ziel eines solchen phasengegliederten Vorgehens ist es, möglichst viele Patienten dauerhaft und rezidivfrei von der allzu oft lebenslangen Erkrankung der chronischen Osteitis zu befreien.

\section{Methodik}

Phase 1: In der ersten Phase steht die Infektsanierung im Vordergrund. Wichtigste Elemente hierbei sind eine vollständige Entfernung aller noch einliegenden Implantate und Fremdkörper und ein radikales chirurgisches Debridement. Unsere chirurgischen Resektionen lehnen sich hierbei weitgehend an die Konzepte der Knochentumorchirurgie an. Die Osteotomien erfolgen orthogonal zur Hauptachse der langen Röhrenknochen weit im gesunden.

Das vom Infekt betroffene Gewebe (Knochen, Faszien, Muskeln, Sehnen, Haut, ggf. unter Miteinbeziehung der Fistel) wird möglichst radikal en bloc reseziert (Abb.1-3). Die zurückbleibenden, unter Umständen sehr ausgedehnten knöchernen Defekte müssen zur Gewährleistung einer Lagerungs- und Übungsstabilität der betroffenen Extremität mit einer Fixateur-Externe-Montage überbrückt werden (Abb.4-5). Der Verschluss des Haut-/Weichteildefektes erfolgt in Phase 1 mit Okklusionsverbänden. Alle 5-7
Tage finden nun in kurzen Narkosen (10-15 min) programmierte Etappenlavagen statt. Dabei wird jeweils vorher vom proximalen und distalen knöchernen Resektionsrand Gewebe für die mikrobiologische Untersuchung entnommen. Es schließt sich eine lokale Spülung mit der Jet-Lavage (3-5 Liter Ringer-Lösung) an. Ob die lokale Anwendung von antiseptischen Lösungen (z.B. Lavasept ${ }^{\circledR}$ ) $\mathrm{zu}$ besseren Resultaten, insbesondere zu einer schnelleren Keimfreiheit des Situs führt, ist nicht gesichert. Sobald der oder die verursachenden Keime isoliert sind, sollte eine testgerechte lokale und systemische Antibiose adjuvant durchgeführt werden. Dies erfolgt auch unter der Vorstellung, dass durch die mechanischen Resektions- und Reinigungsmanöver eine systemische Keimverschleppung vermieden werden soll. Die Einlage von Schwämmen mit Drainagen (Coldex ${ }^{\circledR}$ ) und die Vakuumversiegelung der Wundhöhle komplettieren eine solche Etappenlavage (Abb.6).

Zielpunkt von Phase 1 ist eine keimfreie Defektsituation. Wir definieren dieses Ziel als dann erreicht, wenn drei aufeinanderfolgende Etappen-Revisionen bakteriologisch negative Gewebeproben ergeben. In unserem Krankengut ist dies nach durchschnittlich 6 Revisionen der Fall.

Phase 2: Das von uns propagierte, phasengegliederte Behandlungskonzept der chronischen Osteitis erfordert eine enge interdisziplinäre Zusammenarbeit zwischen Unfallchirurgie und Plastischer Chirurgie. Das Haut-/Weichteilmanagement gelingt auf Grund der zur Sicherung der Radikalität erforderlichen, oft großen Defekte nur in den seltensten Fällen durch einen einfachen sekundären Wundverschluss. Deshalb kommen verschiedene Methoden der lokalen (Abb.7) und freien Lappenplastiken (Abb. 8) zur Anwendung (Tab.1). 

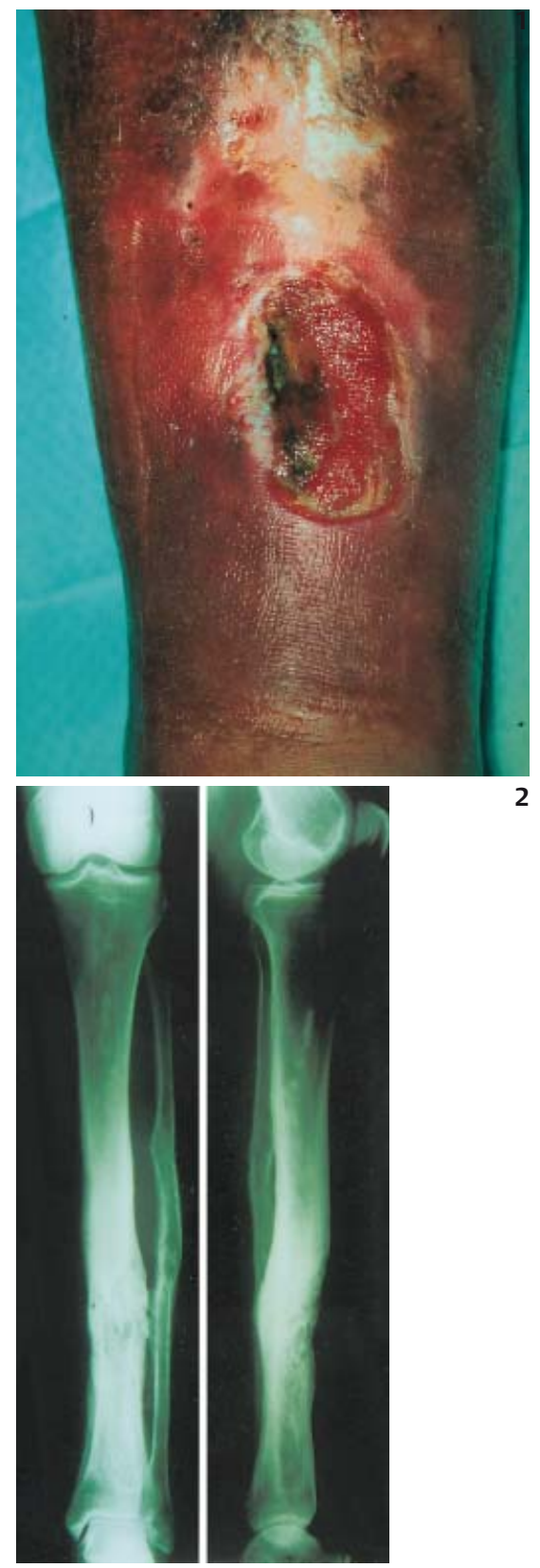

Abb.1 u. 2 46-jähriger Patient. Z. n. III.-gradig offener Unterschenkelfraktur li. beim Fallschirmspringen vor 25 Jahren. Seither chronisch fistelnde Osteitis am li. Unterschenkel.

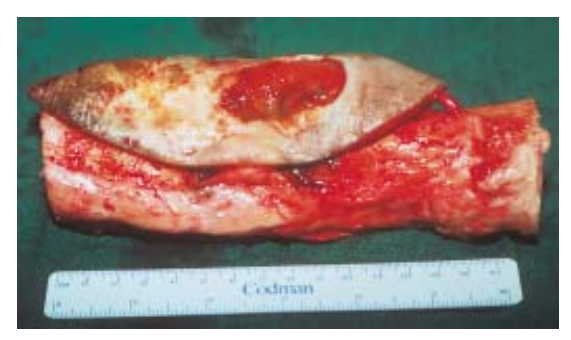

Abb. 3 En-Bloc-Resektion von $17 \mathrm{~cm}$ li. Tibia unter Miteinbeziehung des Fistelsystems durch den Haut-/Weichteilmantel.

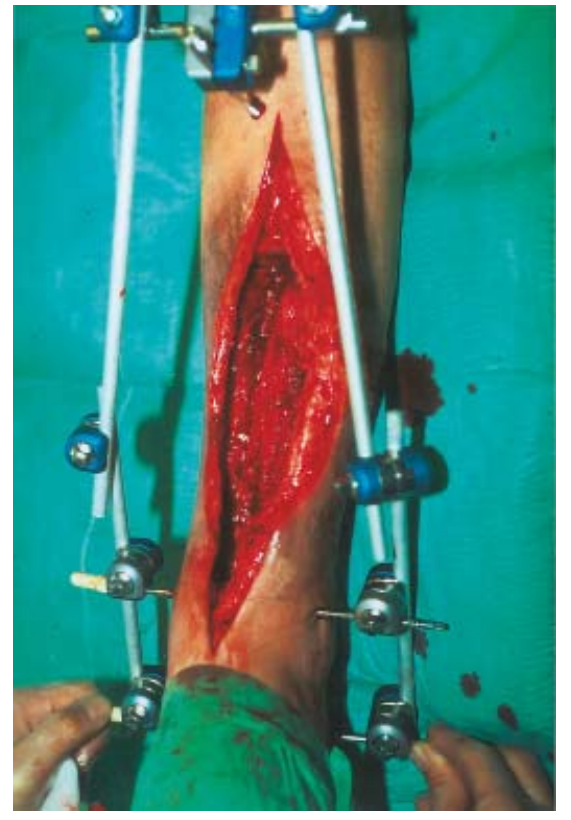

Abb. 4 u. 5 Pat. wie Abb.1 Z.n. En-Bloc-Resektion des infekttragenden Abschnittes und Debridement. Stabilisation defektüberbrükkend mit 3D-Fixateur-Externe-Montage.
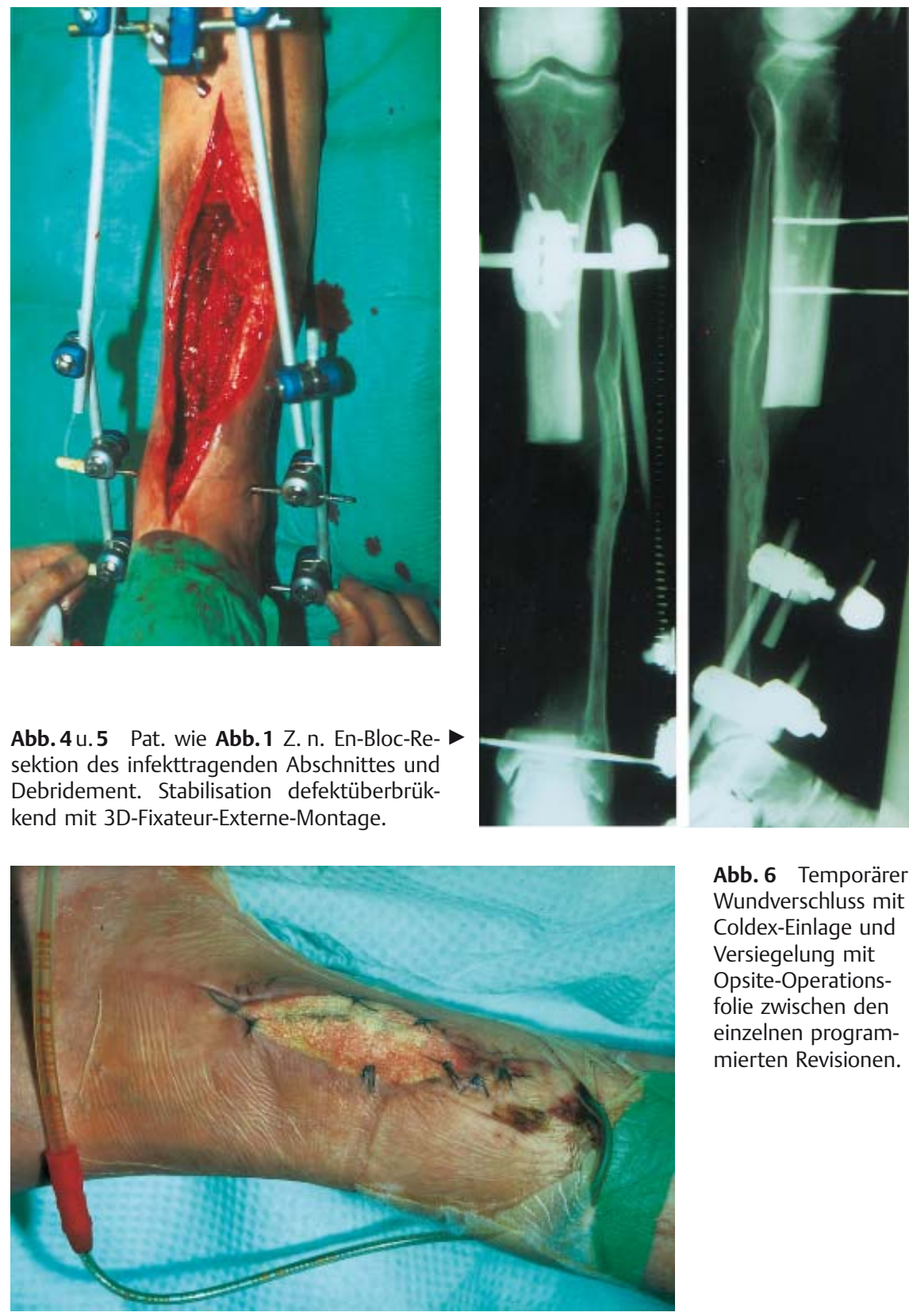

Abb. 6 Temporärer Wundverschluss mit Coldex-Einlage und Versiegelung mit Opsite-Operationsfolie zwischen den einzelnen programmierten Revisionen.

Tab. 1 Lokale und freie Lappenplastiken zur Defektdeckung nach Infekt-Osteotomie und Resektion

Lokal: Gastrocnemiuslappen Soleuslappen Peronaeus-Brevis-Lappen Gracilislappen

Frei: M. Latissimus-Dorsi-Lappen Parascapularlappen

M. Rectus-Abdominis-Lappen

Zielpunkt der Behandlung in Phase 2 ist die langfristige Sicherung der Keimfreiheit der Osteotomieränder und des De- fektes durch eine stabil eingeheilte Haut-/Weichteildeckung. Der Start für Phase 3, die Rekonstruktion der knöchernen Defektstrecke hängt von lokalen und systemischen Parametern in gleicher Weise ab. Das Fehlen lokaler Entzündungszeichen über dem plastisch gedeckten Areal ist ebenso wichtig wie die Beachtung klinischer (subfribrile Temperaturen) und laborchemischer (BSG, Leukos, CRP) Parameter.

In bestimmten, besonders gelagerten Fällen lassen sich Phase 2 und Phase 3 auch kombinieren. Wenn zur Erzielung von 


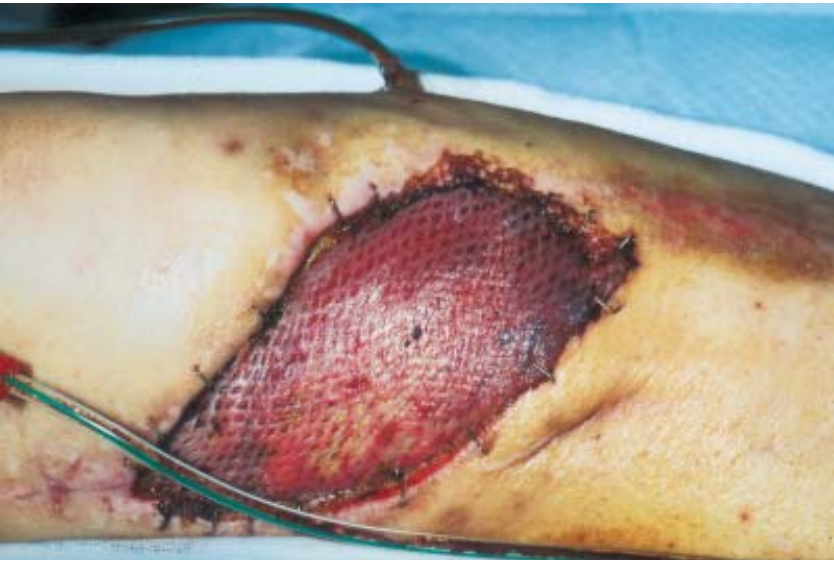

Abb. 7 Beispiel für Haut-/Weichteilmanagement durch lokale Lappenplastik: Defektdeckung an einem Unterschenkel mit medialem Gastrocnemiuslappen und Spalthauttransplantation.

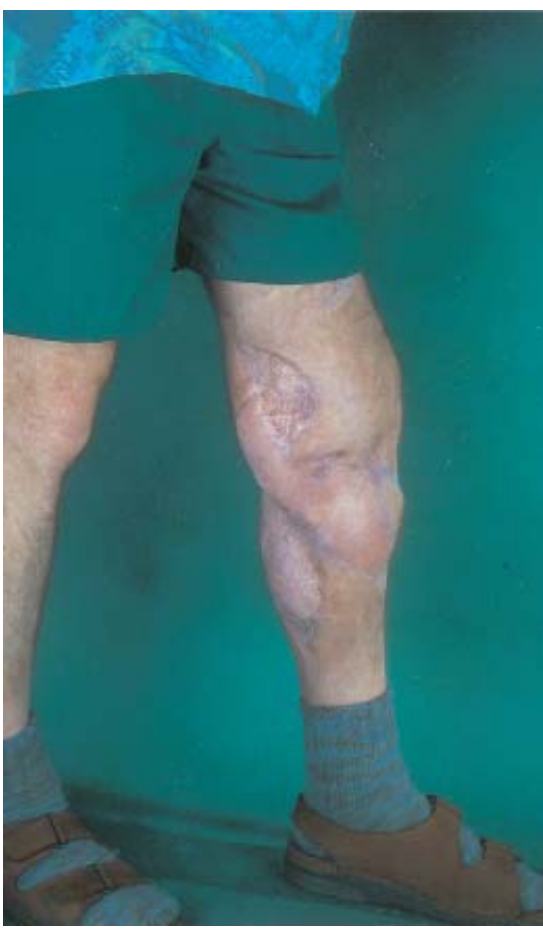

Abb. 8 Beispiel für ein Haut-/Weichteilmanagement mit freier Lappenplastik: Deckung eines ausgedehnten Haut-/Weichteildefektes am li. Ober- und Unterschenkel durch gelenkübergreifende Lappenplastik mit LatissimusDorsi-Transplantat und Mashgraft-Deckung.

Keimfreiheit keine radikale Segmentresektion über mehrere Zentimeter erforderlich war, wenn eine keimfreie, durchblutete knöcherne Brücke („Halbrohr“, „Drittelrohr“) eine verbleibende mechanische Reststabilität (lagerungsstabil) gewährleistet, kann der Defekt auch mit einem osteo-myo-kutanen Lappentransplantat gedeckt werden. Eine additive autologe Spongiosaplastik dient der weiteren biologischen Defektversiegelung

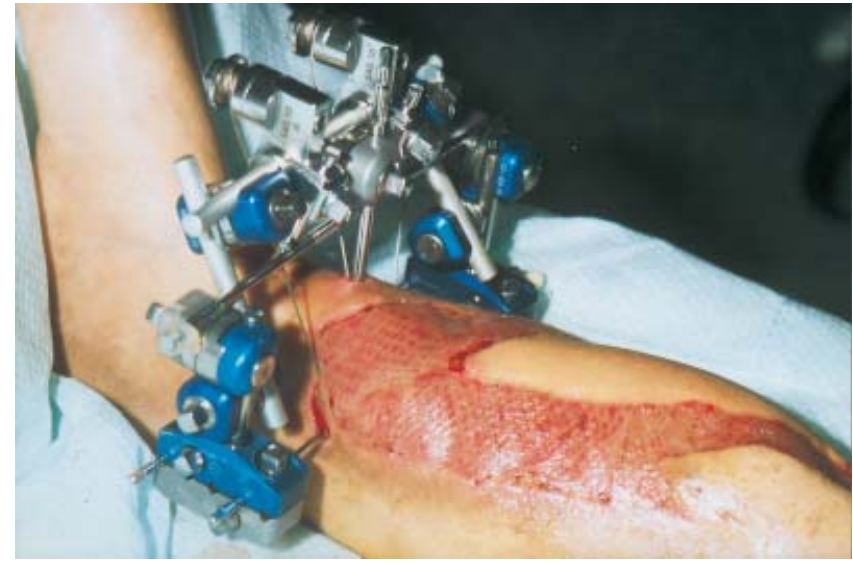

Abb.10 Kallusdistraktion an einem Unterschenkel. Der dargestellte Fixateur externe erfüllt zwei Aufgaben:

1. distale zweifache Verriegelung des einliegenden Marknagels (Fixationssystem)

2. Montage des Seilzugs (Transportsystem)

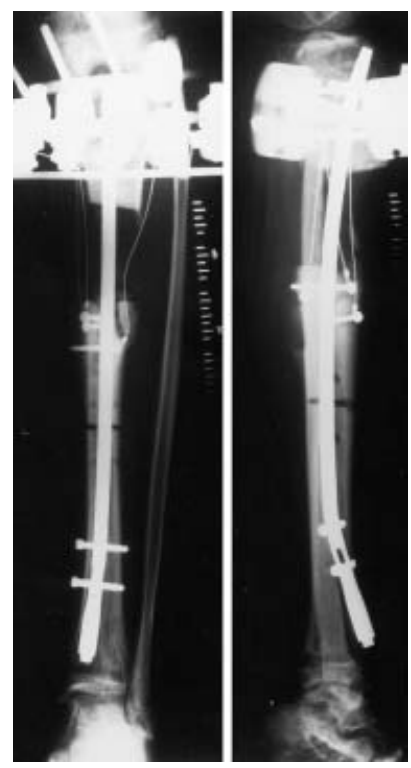

Abb.9 Segmenttransport zur knöchernen Defektüberbrückung mit Kombination aus retrograd eingebrachtem Tibiamarknagel (Fixationssystem) und über Fixateur externe montiertem Seilzugsystem (Transportsystem). Situation unmittelbar nach Osteotomie in der Ruhephase. Transport noch nicht begonnen.

und Wiederherstellung der knöchernen Kontinuität.

Phase 3: Zur Überbrückung des knöchernen Substanzdefektes können verschiedene Verfahren zur Anwendung kommen (Tab. 2).

Die autologe Spongiosaplastik gilt nach wie vor als Standard, muss aber sinnvollerweise auf relativ kurze Defektstrecken

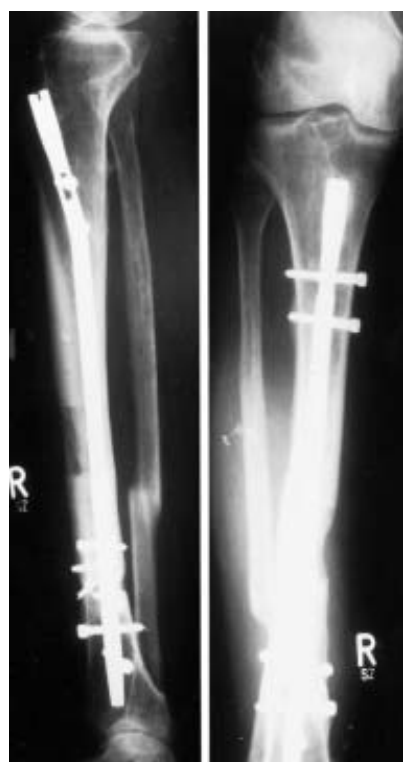

Abb.11 Gleicher Fall wie Abb.10. Nach Abschluss des Transportes ist der Fixateur externe entfernt. Der einliegende Marknagel ist mit Schrauben verriegelt und das Transportsegment ist an der Andockstelle durch eine zusätzliche Drahtcerclage fixiert. Fortschreitende knöcherne Durchbauung des Distraktates.

Tab. 2 Ersatzverfahren zur Überbrückung knöcherner Substanzdefekte

\footnotetext{
- autologe Spongiosatransplantation

- mikrovaskuläre, autologe Knochentransplantation (Fibula, Rippe, Beckenkamm

- Umkehrplastiken

- vaskularisierte, allogene Transplantationen

- Segmenttransport über Kallusdistraktion
} 


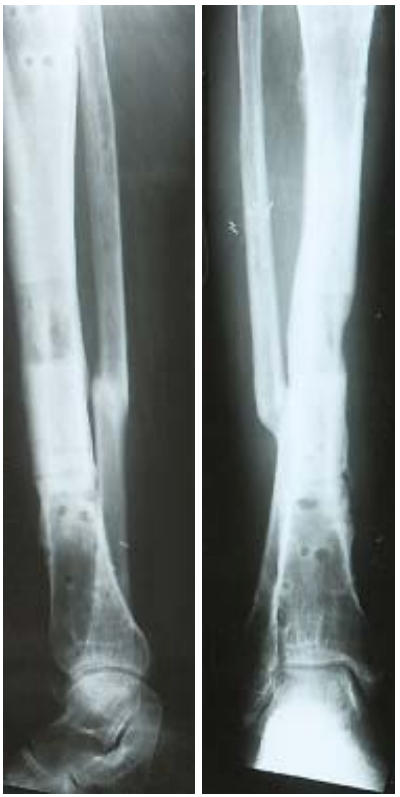

Abb.12 Gleicher Fall wie in Abb.11. Nach weitgehender knöcherner Konsolidierung von Andockzone und Distraktat jetzt nach Entfernung des Marknagels.

(unter $4 \mathrm{~cm}$ ) beschränkt bleiben (Ausnahme: als additive Maßnahme).

Mikrovaskuläre, autologe Knochentransplantationen ermöglichen vielfach nur einen sehr verzögerten Belastungsaufbau. Die Entnahmemorbidität der autologen Transplantate kommt hinzu. Umkehrplastiken und vaskularisierte allogene Transplantate (Immunsuppression!) sind nur in äußersten Ausnahmefällen indiziert. Das „Arbeitspferd“ für den Wiederaufbau langstreckiger knöcherner Defekte nach Osteitis-Resektion ist der Segmenttransport über Kallusdistraktion. Hierzu stehen bekanntlich verschiedene Verfahren zur Verfügung. Die verschiedenen Fixations- und Transportsysteme basieren im wesentlichen auf Ringfixateuren, monolateralen Fixateuren, Marknägeln und Seilzügen.

Wann immer möglich, bevorzugen wir für den Segmenttransport eine Kombi- nation aus Marknagel (Fixationssystem) mit einem Seilzug (Transportsystem) (Abb.9). Die Vorteile dieses Verfahrens liegen im höheren Patientenkomfort, in der erleichterten Physiotherapie und im Fehlen der bei allen Fixateur-ExterneMontage gefürchteten Pin-Infektionen. Die Hauptgefahr des intramedullären Verfahrens besteht zweifelsfrei in der möglichen Reaktivierung der Osteitis im Markraum des Röhrenknochens.

Je nach topographisch-anatomischen Gegebenheiten erfolgt der Segmenttransport auf dem Marknagel als Schiene in anterograder oder retrograder Richtung. Die Osteotomie des Transportsegmentes kann am Oberschenkel idealerweise mit Hilfe einer sogenannten Innensäge erfolgen, für den Unterschenkel liegt ein solches Instrument noch nicht vor. Hier muss die Osteotomie möglichst periostschonend vor der Implantation des Marknagels mit einem Meißel erfolgen. Die Transportgeschwindigkeit liegt beim System Marknagel und Seilzug etwa 10\% über der von monolateralen und Ringfixateur-Systemen. Nach Abschluss des Segmenttransportes können alle externen Implantate des Zugsystems (Fixateur externe, Seilzug) (Abb.10) entfernt werden. Die Ausbehandlung über Neutralisations- und Dynamisierungsphase bis zur knöchernen Konsolidierung des Regenerates und der Andockstelle erfolgt dann ausschließlich über das intramedulläre Implantat (Abb.11). Die Materialentfernung darf nicht zu früh erfolgen. Erst bei weit fortgeschrittener knöcherner Konsolidierung des Regenerates besteht keine Gefahr des sekundären Längenverlustes mehr (Abb.12).

\section{Schlussfolgerung}

Die Korrektur von Fehlstellungen, denen eine vorausgegangene - jetzt latente oder noch immer aktive - Knocheninfektion zu Grunde liegt, wird bez. des Vorgehens im Wesentlichen durch das Infektionsmanagement bestimmt. Nach jahr (zehnt)elangem kompliziertem Krankheitsverlauf liegen oft derart kritische Weichteilverhältnisse vor, dass darüberhinaus eine enge interdisziplinäre $\mathrm{Zu}$ sammenarbeit zwischen Unfallchirurgie und Plastischer Chirurgie erforderlich ist. Korrigierende Osteotomien fehlstellungs- und infektfern durchzuführen, ohne gleichzeitig den Focus zu sanieren, lehnen wir ab. Die Beherrschung der chronischen Knochen + Haut-/Weichteilinfektion besitzt für uns absolute Priorität. Deshalb muss es das Ziel sein,

- Korrekturmaßnahmen und

- Infektsanierung

mit ein und demselben Verfahren durchzuführen.

Ein systematisches und phasenorientiertes Therapiekonzept ermöglicht in unserem Krankengut in ca. 85\% der Fälle eine dauerhaft rezidivfreie Behandlung der chronischen Osteitis. Allerdings muss dennoch in $15 \%$ trotz radikaler Resektion mit einem Osteitisrezidiv gerechnet werden. Nach wie vor erweist sich dann bei der Mehrzahl dieser Rezidivfälle die Amputation der betroffenen Extremität oftmals als der einzige Ausweg, einen Patienten medizinisch und sozial zu rehabilitieren.

Prof. Dr. med. Dr. rer. nat. G. O. Hofmann Chirurg/Unfallchirurg, Oberarzt

BG-Unfallklinik Murnau

Prof.-Küscher-Str. 8

82418 Murnau 